\title{
Arrhythmias and symptoms during treadmill testing three weeks after myocardial infarction in 100 patients
}

\author{
Martin Ericsson, Arne Granath, Paul Ohlsén, Tore Södermark, and Ulderico Volpe \\ From the Departments of Clinical Physiology and Internal Medicine, \\ Danderyd Hospital, S-182 o3 Danderyd, Sweden
}

Treadmill tests with continuous electrocardiographic recording were carried out 3 weeks after the acute episode began on 100 patients with myocardial infarction. Ventricular premature beats were recorded in 3 patients at rest immediately before the test, and in I9 during and/or after the test. The test led to a change of treatment for I6 patients. No severe arrhythmias or reinfarctions were induced by the test. Twenty patients developed angina pectoris. The incidence of ventricular premature beats during the test was higher in the patients with arrhythmia during the acute phase (10/37) than in those without $(7 / 55)$. This difference is not statistically significant. The treadmill test proved to be a sensitive method for demonstrating a tendency to arrhythmia in patients with recent infarction. It permits observation of the patient during exercise similar to that performed during rehabilitation.

The mortality rate of patients with myocardial infarction during the first year after discharge from hospital is said to be approximately 8 per cent (Pell and D'Alonzo, 1964). Many die suddenly, probably because of ventricular arrhythmia (Lown, Kosowsky, and Klein, 1969). Mortality in patients with coronary heart disease appears to be greater in patients with ventricular premature beats than in those without (Chiang et al., 1969).

A two-year follow-up of men who had suffered myocardial infarction disclosed a mortality twice as high for patients with frequent ventricular premature beats in a single electrocardiogram than for those with rare or no ventricular premature beats. The difference was significant even after correction for coronary heart disease risk factors (Lown and Wolf, 1971). The discovery of a tendency to ventricular arrhythmia soon after infarction would be a help in the choice of treatment. Ventricular arrhythmia is more likely to be detected during brief, moderately heavy exercise than during continuous ambulatory monitoring for two hours (Kosowsky et al., I97I). Therefore, we arranged for patients with acute myocardial infarction to perform a standardized functional test on a treadmill, immediately before planned discharge. The test was also intended

Received 27 December 1972. to assist in evaluating the patient's functional state as a means of arriving at individualized programmes of management.

\section{Subjects}

The study comprises roo consecutive exercise tests on a treadmill performed by patients with acute myocardial infarction in the period April 1970 to October 1971. There were 97 patients altogether ( 90 men and 7 women), 3 being examined a second time because of reinfarction. All were treated in the hospital's coronary care unit which serves a population of 180,000 . The tests were performed immediately before the planned discharge from hospital, i.e. a mean of 21 days after the acute episode began. A total of 228 patients with infarction (I74 men and 54 women) attended the unit during the period in question. Of these, 44 died during their stay in the hospital and 95 of the remaining 184 were older than 65 years. Nobody above this age was tested on the treadmill during the first half of the study, owing to a lack of resources, and only 16 during the second half. Of the remaining patients under 65,8 were not tested. A lack of beds in the unit made it necessary to move 5 of these to another ward during the second week and the other 3 had complicating disorders (hemiplegia, fracture of the neck of the femur, and malignancy, respectively).

The distribution by age and sex for the Ioo patients in the study is shown in Fig. I (the 3 patients with 2 infarction episodes are counted as 6 patients). Seventy- 


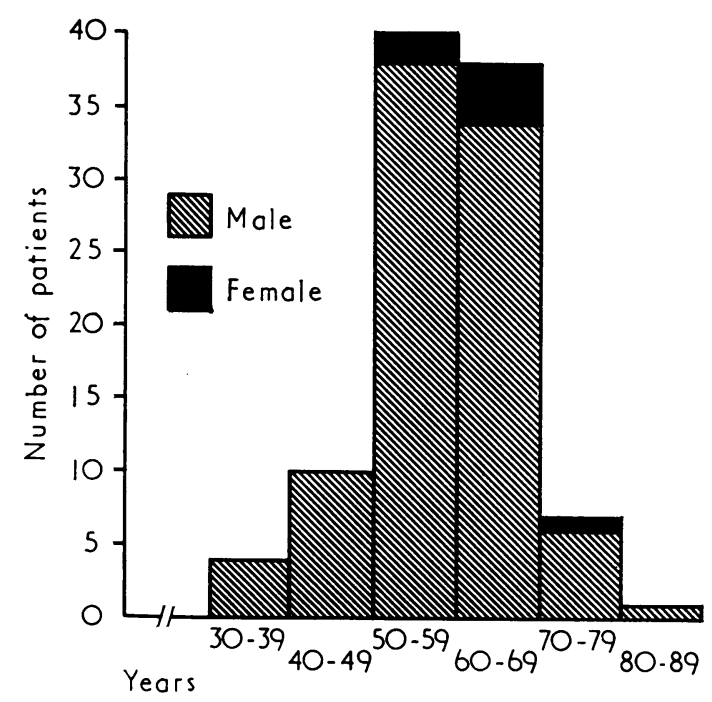

FIG. I The age and sex of the Ioo patients.

five cases were evaluated as first infarctions, 20 as second, 3 as third, and 2 as fourth. The maximum values for serum aspartate aminotransferase (IU) for 93 patients are shown in Fig. 2 (7 patients were admitted more than 3 days after the onset of symptoms). Heart volume was determined radiologically in a conventional manner

TABLE I Site of myocardial infarctions in 94 patients

\begin{tabular}{lc}
\hline Location & No. \\
\hline Anteroseptal/anterolateral & 50 \\
Diaphragmatic & 36 \\
Posterior & 6 \\
Anteroseptal/anterolateral + diaphragmatic & I \\
Diaphragmatic + posterior & I \\
\hline
\end{tabular}

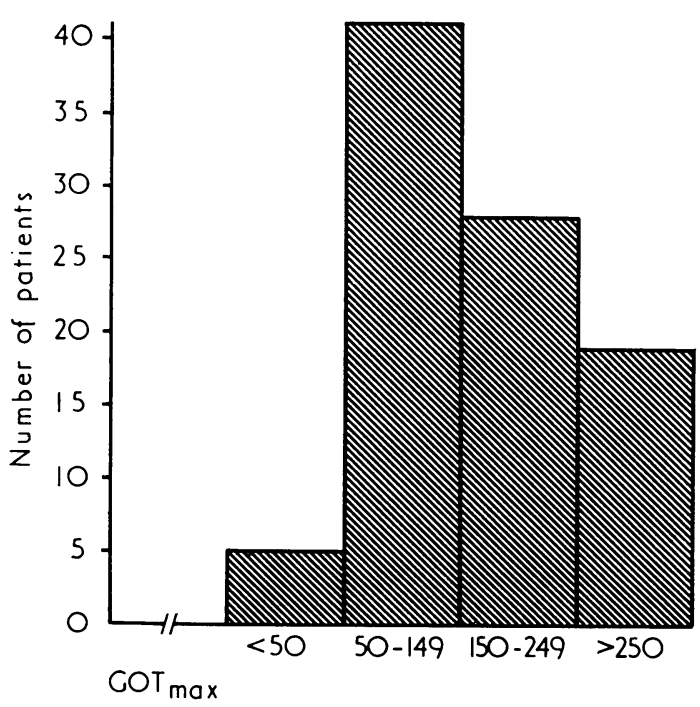

FIG. 2 Maximum aspartate aminotransferase in 93 patients.

with the patient upright, using the formula of Jonsell (1939). The relative heart volume was more than 500 $\mathrm{ml} / \mathrm{m}^{2}$ body surface area in 49 men and more than 450 $\mathrm{ml} / \mathrm{m}^{2}$ in 4 women. No one had a heart volume greater than $750 \mathrm{ml} / \mathrm{m}^{2}$.

The sites of infarction according to the electrocardiogram are shown in Table $\mathrm{I}$. A definite site could be identified in 94 patients. Of the remaining 6,3 had had previous myocardial infarction, one displayed transient ST depression corresponding to the anterior wall as the only electrocardiographic change, one had left bundlebranch block, and one had non-specific electrocardiographic changes.

A minimum of 2 of the following 3 criteria were required for diagnosis.

I) Acute central chest pain, lasting more than 30 minutes.

2) A rise of aspartate aminotransferase with values

TABLE 2 Ventricular arrhythmias during monitoring day I-3, during rest, and before, during, and after treadmill test

\begin{tabular}{|c|c|c|c|c|c|c|}
\hline & \multicolumn{4}{|c|}{ No. of patients with ventricular premature beats } & \multirow{3}{*}{$\begin{array}{l}\text { Ventricular } \\
\text { fibrillation }\end{array}$} & \multirow{3}{*}{$\begin{array}{l}\text { Total no. of } \\
\text { patients with } \\
\text { ventricular } \\
\text { arrhythmias }\end{array}$} \\
\hline & \multicolumn{2}{|l|}{$\geq 5 / \min$} & \multicolumn{2}{|c|}{ Series } & & \\
\hline & Unifocal & Multifocal & $2-3$ & $\geq 4$ & & \\
\hline $\begin{array}{l}\text { During monitoring } \\
\text { During rest before treadmill test } \\
\text { During and after treadmill test }\end{array}$ & $\begin{array}{r}\text { II } \\
2 \\
6\end{array}$ & $\begin{array}{l}3 \\
1 \\
8\end{array}$ & $\frac{24}{7}$ & $\frac{8}{1}$ & $\frac{1}{-}$ & $\begin{array}{r}37 \\
3 \\
19\end{array}$ \\
\hline
\end{tabular}


higher than those of alanine aminotransferase (in International Units, IU). Serum lactic dehydrogenase (IU) isozyme assay helped to confirm the diagnosis in a few cases in which the aspartate aminotransferase was only slightly raised.

3) Electrocardiographic pattern consistent with infarction. Posterior localization of the infarction was confirmed by vectorcardiogram (Frank system).

The patients were mobilized during their hospital stay with the aid of a physical therapist: the programme allowed for visits to the lavatory from the second or third day and an ambulatory regimen from the seventh to the tenth day. In the third week patients were allowed to climb stairs. Mobilization proceeded more slowly for 7 patients with serious arrhythmia and/or myocardial insufficiency.

\section{Methods}

All patients were examined before discharge from hospital, with treadmill tests at a walking speed of 0.5 $\mathrm{m} / \mathrm{sec}$. The mechanical work was derived from body weight, velocity, and the angle of inclination of the treadmill by the use of a nomogram. (Mechanical work in $\mathrm{kpm} / \mathrm{min}=$ sine $\alpha \times 60 \times \mathrm{a} \times \mathrm{b} . \alpha=$ angle of inclination in degrees, $a=$ treadmill velocity in $\mathrm{m} / \mathrm{sec}, \mathrm{b}=$ body weight in kg.)

Usually the first load was 100 or $200 \mathrm{kpm} / \mathrm{min}$ (approximately 16 or $33 \mathrm{Watt}$, respectively), depending on age, sex, and clinical condition. The intention was to achieve a maximal heart rate of approximately I 20 beats/ min. If the heart rate after 4 to 6 minutes on the treadmill was below I 20 beats $/ \mathrm{min}$ and the patient had no distress, he was allowed to continue at a higher load.

The treadmill was a standard Collins (P2000) I-8 NPH, fitted with an angle measuring device which permitted settings with an accuracy of $1 / 6^{\circ}$, and a calibrated speedometer with an accuracy of $0.10 \mathrm{~m} / \mathrm{sec}$. With a constant velocity and the angles used in the study oxygen consumption in a healthy subject displays an approximately linear relation to the angle of inclination and therefore to the mechanical work (Bobbert, 1960). This should also apply to a coronary patient. When comparing individuals with different body weights, however, mechanical work is a better measure of the circulatory demands imposed by exercise than the angle of inclination and treadmill velocity alone, the latter being conventional measures.

The electrocardiogram was recorded at recumbent rest for I minute, in a standing position before the test, and continuously during and for the first ro minutes after exercise. Post-exercise recordings were made with the patient recumbent. During exercise, chest-head leads $\left(\mathrm{CH}_{1}, 2,3,4,5,7\right)$ were used with the reference electrode on the forehead. Before and after exercise, the corresponding chest-right arm leads $\left(C_{1}, 2,3,4,5,7\right)$ were used in addition to extremity leads.

A 6-channel direct-writing Mingograf 8I electrocardiogram (Elema-Schönander $\mathrm{AB}$ ) was used for the recordings. The electrocardiogram was monitored continuously on an oscilloscope throughout the examination.
The functional test was stopped in the event of the following occurring: ( $\mathrm{I}$ ) angina pectoris or dyspnoea; (2) frequent ventricular premature beats; (3) heart rate exceeding I 40 beats/minute.

The functional test was continuously supervised by a physician.

\section{Results}

The test was ended at a work load of $100 \mathrm{kpm} / \mathrm{min}$ in II patients, at $200 \mathrm{kpm} / \mathrm{min}$ in 59 , at $300 \mathrm{kpm} /$ $\mathrm{min}$ in 5 , and at $400 \mathrm{kpm} / \mathrm{min}$ in 23 ; I patient continued to $500 \mathrm{kpm} / \mathrm{min}$ and another to $600 \mathrm{kpm} / \mathrm{min}$. The average duration of exercise at the final load was five minutes. The mean maximal heart rate during the test was $118 / \mathrm{min}$. Twenty patients developed angina pectoris, and 5 pronounced dyspnoea and/or fatigue. All complaints had disappeared to minutes after the conclusion of exercise. The incidence of ventricular premature beats during the acute stage and during the treadmill test is shown in Table 2. No severe arrhythmias or reinfarctions were induced by the test.

Ventricular premature beats ( 5 or more per minute or a series of at least 2) were recorded in only 3 patients at rest immediately before the test and in I9 during or within Io minutes after exercise. Of the 3 patients with premature beats at rest, 2 were included in the 19. Of these 19 patients, 6 were on antiarrhythmic therapy.

Of the 92 patients continuously monitored during the first two to three days of the acute phase, 37 had either 5 or more ventricular premature beats a minute or a series of at least 2 . Of these 37 patients, Io also had this arrhythmia during the functional test; five of them were on antiarrhythmic therapy. Of the 55 patients without ventricular arrhythmia during the acute phase, only 7 displayed ventricular premature beats during the functional test; one of them was on antiarrhythmic treatment. Thus the incidence of ventricular premature beats during the treadmill test was higher in the patients with arrhythmia during the acute phase (10/37) than in those without (7/55). This difference is not statistically significant.

At the time of the test antiarrhythmic therapy (procainamide $0.5 \mathrm{~g}$ four times daily in most cases) was given to 16 of the 37 patients in the former group $(43 \%)$ and only 7 of the $55(13 \%)$ in the latter. Sixty-one patients were being treated with digitalis and/or diuretics. The test led to a change in the drug treatment of 16 patients, most frequently to the initiation of antiarrhythmic therapy (I I patients). Eight patients had their hospital stay prolonged and were retested. One patient performed 3 treadmill tests and another patient 4 before discharge.

All patients were followed up for a minimum of 
three months after the onset of symptoms. During this period, 5 patients died, 4 of them suddenly and I after reinfarction with cardiogenic shock. Of the 4 patients who died suddenly, 2 had had ventricular premature beats during the treadmill test; in one of them the beats had been frequent and multifocal during 4 tests performed before discharge, despite the use of various antiarrhythmic drugs. The other 2 patients who died suddenly had had no arrhythmia during the treadmill test. One of them had developed angina pectoris and the test was stopped after 4 minutes at $200 \mathrm{kpm} / \mathrm{min}$; this patient died in the shower immediately before discharge some days after the test. The other had to discontinue the test because of a high heart rate (137 beats/min at 200 $\mathrm{kpm} / \mathrm{min}$ ) and mild dyspnoea. Of the 5 patients who died in these 3 months, 4 had cardiomegaly and 3 had had angina pectoris during the treadmill test.

During exercise a positive $T$ developed in the electrocardiogram in 24 patients who had an inverted $T$ at rest. The $S T$ segment was raised above the baseline in 44 patients by a mean of $0.18 \mathrm{mV}$ in leads reflecting the infarcted area. In the patients with diaphragmatic infarction, such repolarization changes could not be recorded until after work, since extremity leads were not used during exercise. Fifty-nine patients had one or both of these electrocardiographic abnormalities. Similar abnormalities during exercise have been reported by Atterhög, Ekelund, and Kaijser (197I).

\section{Discussion}

This study shows that a tendency to arrhythmia soon after an acute myocardial infarction can be demonstrated with treadmill tests more frequently than with routine electrocardiograms at rest. Moreover, the test made it easier to determine the factors limiting the patient's work capacity. There were no indications that the procedure described involved any risks. We could not find any published reports about the use of standardized exercise tests for the detection of arrhythmia in this early phase after myocardial infarction.

At three-month follow-up the mortality was higher in the group of patients with ventricular premature beats at the treadmill test $(2 / 19)$ than in that without (2/8I). To evaluate the importance of this finding we plan further studies.

Treadmill tests provide an opportunity of observing the patient during exercise similar to that to be prescribed for rehabilitation.

\section{References}

Atterhög, J. H., Ekelund, L. G., and Kaijser, L. (I97I). Electrocardiographic abnormalities during exercise 3 weeks to 18 months after anterior myocardial infarction. British Heart fournal, 33, 87I.

Bobbert, A. C. (1960). Energy expenditure in level and grade walking. Fournal of Applied Physiology, 15, 1015.

Chiang, B. N., Perlman, L. V., Ostrander, L. D., Jr., and Epstein, F. H. (1969). Relationship of premature systoles to coronary heart disease and sudden death in the Tecumseh epidemiologic study. Annals of Internal Medicine, 70, II59.

Jonsell, S. (1939). A method for determination of the heart size by teleroentgenography. Acta Radiologica, 20, 325.

Kosowsky, B. D., Lown, B., Whiting, R., and Guiney, T. (197I). Occurrence of ventricular arrhythmias with exercise as compared to monitoring. Circulation, 44, 826.

Lown, B., Kosowsky, B. D., and Klein, M. D. (1969). Pathogenesis, prevention, and treatment of arrhythmias in myocardial infarction. Circulation, 40, Suppl. IV, 26r.

Lown, B., and Wolf, M. (197I). Approaches to sudden death from coronary heart disease. Circulation, 44, 130.

Pell, S., and D'Alonzo, C. A. (1964). Immediate mortality and five-year survival of employed men with a first myocardial infarction. New England fournal of Medicine, 270, 915 .

Requests for reprints to Dr. Arne Granath, Department of Clinical Physiology, Danderyd Hospital, S-182 03 Danderyd, Sweden. 